\title{
A concept of a robust solution of a multicriterial linear programming problem
}

\author{
Dorota Kuchta
}

Published online: 9 November 2010

(C) The Author(s) 2010. This article is published with open access at Springerlink.com

\begin{abstract}
A new concept of a robust solution of a multicriterial linear programming problem is proposed. The robust solution is understood here as the best starting point, prepared while the preferences of the decision maker with respect to the criteria are still unknown, for the adaptation of the solution to the preferences of the decision maker, once they are finally known. The objective is the total cost of the initial preparation and of the later potential adaptation of the solution. In the starting robust solution the decision variables may have interval values. The problem can be solved by means of the simplex algorithm. A numerical example illustrates the approach.
\end{abstract}

Keywords Multicriteria programming $\cdot$ Robust solution

\section{Introduction}

In recent years the robust approach to optimization problems has been gaining more and more attention in the operations research. This notion of robustness is quite close to that of stability. Both notions are very important because of the instability of the environment in which decisions are taken. In the moment they are taken not all the information about their future implementation conditions is available, thus the question arises: will the decision we have taken now, the solution we have selected, be of some worth in the future?

There are many approaches to the robustness (stability) of a solution of a decision problem. Very often the question is asked for which set of problem parameters the solution we have determined now will remain "good" in the future (the notion

D. Kuchta $(\bowtie)$

Institute of Organisation and Management, Wroclaw University of Technology,

U1. Smoluchowskiego 25, 50-371 Wroclaw, Poland

e-mail: dorota.kuchta@pwr.wroc.pl 
"good" may mean "optimal", "close to optimal", etc.)—see e.g. El-Banna and Zarea (2001) or Saad (1995). In many approaches all the possible scenarios are assumed to be known and we are looking for a solution which will be good even if the worst scenario takes place or which will assure us the minimal regret of not having chosen another solution (e.g. Kouvelis and Yu 1997). The notion of the worst scenario is weakened in Ben-Tal and Nemirovsky (1999) — the authors assume not the very worst scenario, but some scenarios close to the worst one-they assume that almost never everything goes bad at the same time. Véronneau and Cimon (2007) propose a holistic approach to robustness - they consider various systems in general and ask how to prevent the systems from collapsing. Kuchta (2010) has implemented this more psychological approach in project scheduling.

Often we have to choose a temporary solution in order to start the preparations (especially in cases when initial preparations take a lot of time and it is not possible to wait with them until everything is known), but we are prepared to change it (i.e. the values of decision variables) if necessary. However, we would like to prepare ourselves in such a way that the cost or effort of the changes to the decision variables will be as small as possible. And this preparation involves a good choice of the starting or temporary solution. This approach to robustness is known in the context of scheduling. In that domain the initial schedule is often only a temporary one and changes constantly in the realization process. The schedule robustness approach (de Vonder et al. 2006) tries to determine an initial schedule whose decision variables (e.g. the start times of individual activities) are bound to change somehow and we want to select them in such a way that the changes to them (which we are ready to implement in the future if necessary) are as small or as cheap as possible.

In this paper we apply this latter approach to multicriteria linear programming problems. To our knowledge, robustness of multicrtieria programming problems is not a widely explored area (it is discussed only in El-Banna and Zarea 2001; Kuchta 2004), and in those papers it is regarded from the formerly mentioned point of view- the solution we have selected will remain the solution to be implemented and we ask only whether this solution will remain good if the parameters of the problem change. Here propose an approach similar to the "schedule robustness" - we are ready to modify later the initial solution, the values of the decision variables, once the uncertainty is not there any more, and we are looking for a reasonably good temporary, starting solution.

There are two completely new elements in our approach. The first new element is the source of uncertainty in the decision making process. In our case it not the value of parameters, like it is in all the robustness/stability problems considered in the literature, but the way the decision maker will finally want to solve the given multiobjective problem. We know which objective functions may be potentially taken into account, we know also what are the possible ways he might choose to treat them (he may choose one of the non-dominated solutions, he may also use a goal programming or a lexicographic or another approach to solving the multiobjective problem see e.g. Steuer 1986) but we do not know yet for which of the possibilities he will decide. We only assume to know all the possibilities which might be chosen. The other new element is that our temporary, starting solution does not have to be a crisp one-we are ready to prepare ourselves to implement a whole spectrum of solutions, thus the initial solution may have interval values of decision variables (although the final one will be 
a crisp one). Of course, such preparations are more expensive than preparing just one crisp solution-however, it may turn out to be less expensive if the cost of adapting the selected interval solution to the one finally desired by the decision maker (once his approach to the multiobjective problem is finally known) is taken onto account. By selecting a temporary interval solution we are better prepared for more possibilities and it may be easier/cheaper to adapt the initial solution to the final one than it would have been the case if the initial solution had to be crisp.

The structure of the paper is as follows: first we formulate the problem, then we show how to determine the robust solution in the sense we are proposing here, and finally we present a simple example illustrating the proposed approach.

\section{Problem formulation}

Let us consider a linear programming problem with I decision variables $x_{i}(i=1$, $\ldots, I$ ), a feasible set $\mathrm{X}$ (a subset of $\mathfrak{R}^{I}$ ) and with $M$ objective functions $f_{m}(m=1, \ldots, M)$ being minimized or maximized and with $\mathrm{J}$ "solution candidates" $\mathbf{x}^{j}(j=1, \ldots, J), \mathbf{x}^{j} \in X$. Each solution candidate corresponds to one way the decision maker might want to solve the multiobjective problem with objective functions $f_{m}(m=1, \ldots, M)$. As mentioned in the introduction, the solution candidates will often belong to the set of nondominated solutions, but may also be selected by using another approach to the multiobjective problems, not necessarily leading to nondominated solutions (like goal programming or lexicographic approaches). We simply have to identify all the possible methods the decision maker might possibly want to use (the number of these methods is I) and determine one solution according to each of them.

In this situation of not knowing exactly what will be the attitude of the decision maker towards the multiobjective problem, we have to make a decision, thus solve the problem at least temporarily, in order to start the preparation work. However, we consider the case where the temporal solution of the problem does not consist in choosing one vector $\mathbf{x}^{0}, \mathbf{x}^{0} \in X$, like it is in the classical case, but-because of the uncertainty of the attitude of the decision maker with respect to the multitude of objectives-we are disposed to prepare our plans (e.g. production and/or sales plans) in a more flexible or broader way, thus in the "interval" form. Thus, we are looking for a solution in the following form: $\left\{\left[\underline{x}_{i}, \underline{x}_{i}+\delta\right]\right\}_{i=1}^{I}$, such that $\left[\underline{x}_{i}, \underline{x}_{i}+\delta\right] \subset X, i=1, \ldots, I$. In other words, we will be prepared to realize any production/sales plan $\mathbf{x}^{0}$ such that $x_{i}^{0} \in\left[\underline{x}_{i}, \underline{x}_{i}+\delta\right], i=1, \ldots, I$. Such a flexible preparation of an "interval" plan entails of course certain cost. We assume this cost will be the higher, the greater $\delta$.

Example: Let us consider the following simple linear programming problem with three objective functions:

$$
\begin{aligned}
& f_{1}\left(x_{1}, x_{2}\right)=x_{2} \rightarrow \max \\
& f_{2}\left(x_{1}, x_{2}\right)=x_{1} \rightarrow \max \\
& f_{3}\left(x_{1}, x_{2}\right)=x_{1}+x_{2} \rightarrow \max \\
& x_{1} \leq 4
\end{aligned}
$$




$$
\begin{aligned}
& x_{2} \leq 4 \\
& x_{1}+x_{2} \leq 6 \\
& x_{1}, x_{2} \geq 0
\end{aligned}
$$

Let us also assume that we think that three attitudes of the decision maker are possible, but we do not know yet which one will occur:

i) $j=1$ : objective functions $f_{1}$ and $f_{2}$ are taken into account in the lexicographic way, where $f_{1}$ will have the highest hierarchy, in this case we assume that solution $\mathbf{x}^{1}=(1 / 2,4)$ would be preferred

ii) $j=2$ : only objective function $f_{3}$ is taken into account, we assume that solution $\mathbf{x}^{2}=(3,3)$ would be preferred

iii) $j=3$ : again the objective functions $f_{1}$ and $f_{2}$ are taken into account also in the lexicographic way, but now $f_{2}$ will have the highest hierarchy, in this case we assume that solution $\mathbf{x}^{3}=(4,1 / 2)$ would be preferred

The following figure shows the set of feasible solutions of problem (1), the three solution candidates (only one of them is non-dominated) and an arbitrary temporary interval solution of the problem —one with the coordinates $\{[2,3],[1.5,2.5]\}\left(\underline{x}_{1}=2\right.$, $\left.\underline{x}_{2}=1.5, \delta=1\right)$.

And then, when the preferences of the decision maker become clear and it will be known which one of the solutions $\mathbf{x}^{j}(j=1, \ldots, J)$ he would like to implement, we may have to adapt our solution to his preferences, thus we may have to make the effort and incur the corresponding cost. We assume this cost will be proportional to, once a solution $\left\{\left[\underline{x}_{i}, \underline{x}_{i}+\delta\right]\right\}_{i=1}^{I}$ is selected and the preferences of the decision maker are known, thus the preferred solution candidate $\mathbf{x}^{j_{0}}\left(j_{0}=1, \ldots, N\right)$ is selected, the value $A C$ (adaptation cost), defined as:

$$
A C\left(\left\{\underline{x}_{i}\right\}_{i=1}^{I}, \mathbf{x}^{j_{0}}, \delta\right)=\min _{x_{i j_{0}} \in \in\left[\underline{x}_{i}, \underline{x}_{i}+\delta\right]} \max _{i=1, \ldots I}\left|x_{i j_{0}}-x_{i}^{j_{0}}\right|
$$

Thus, we assume the adaptation cost is proportional to the maximum of the absolute values of the differences $\left|x_{i j_{0}}-x_{i}^{j_{0}}\right|, i=1, \ldots, I$, where $x_{i}^{j_{0}}, i=1, \ldots, I$ are the components of the preferred solution candidate $\mathbf{x}^{j_{0}}$ and $x_{i j_{0}}, i=1, \ldots, I$ are elements of the intervals $\left\{\left[\underline{x}_{i i}, \underline{x}_{i}+\delta\right]\right\}_{i=1}^{I}$ (thus values of the decision variables which we will be prepared to implement without any cost and effort and which can be taken as the starting points for the adaptation of the solution $\left\{\left[\underline{x}_{i i}, \underline{x}_{i}+\delta\right]\right\}_{i=1}^{I}$ to the desired solution $\mathbf{x}^{j_{0}}$ ), chosen in such a way that $A C$ is as small as possible.

In our example and the situation represented in Fig. 1, we have:

$$
\begin{aligned}
& -A C\left(\{2,1.5\}, \mathbf{x}^{1}, 1\right)=\min _{x_{11} \in \in[2,3], x_{21} \in \in[1.5,2.5]} \max _{i=1,2}\left|x_{i 1}-x_{i}^{1}\right|=1.5 \\
& -A C\left(\{2,1.5\}, \mathbf{x}^{2}, 1\right)=\min _{x_{12} \in \in[2,3], x_{22} \in \in[1.5,2.5]} \max _{i=1,2}\left|x_{i 2}-x_{i}^{2}\right|=0.5 \\
& -A C\left(\{2,1.5\}, \mathbf{x}^{3}, 1\right)=\min _{x_{13} \in \in[2,3], x_{23} \in \in[1.5,2.5]} \max _{i=1,2}\left|x_{i 3}-x_{3}^{1}\right|=1
\end{aligned}
$$




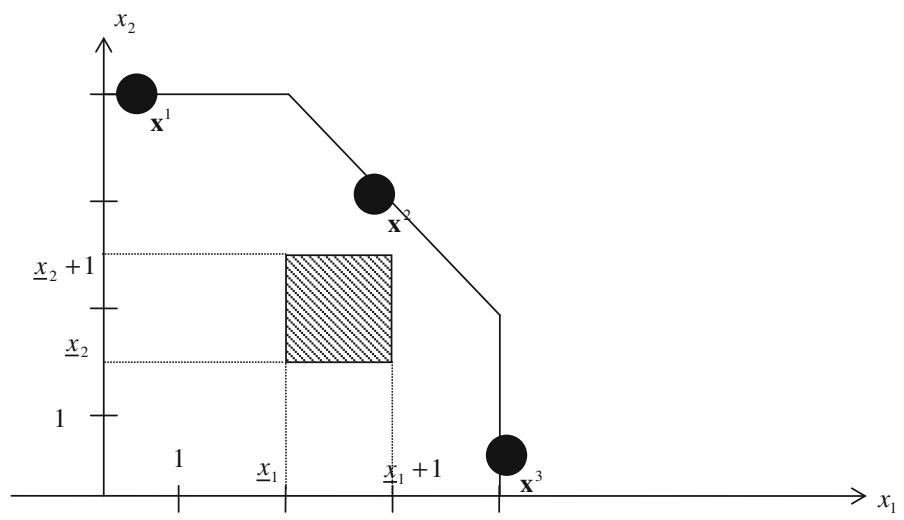

Fig. 1 The set of feasible solutions, the solution candidates and an example of an interval feasible solution for the example problem

This is so, because the starting point for the adaptation of the selected interval solution to the solution candidate $\mathbf{x}^{1}$ is the point $(1,2.5)\left(x_{11}=1, x_{12}=2.5\right)$, and then $\max _{i=1,2}\left|x_{i 1}-x_{i}^{1}\right|=\max \{|2-0.5|,|2.5-4|\}=1.5$. In the analogous way, for the solution candidate $\mathbf{x}^{2}$ the point $(2,2.5)$ is the nearest point (in the sense of the distance we have selected) in the interval solution selected and for this point the adaptation cost is 0.5 . The nearest point from among those corresponding to the plans we are ready to implement without any adaptation cost, with respect to the solution candidate $\mathbf{x}^{3}$, is the point $(3,1.5)$ and thus the adaptation cost in this case would be equal to 1 .

The problem will consist in determining a robust solution $\left\{\left[\underline{x}_{i}, \underline{x}_{i}+\delta\right]\right\}_{i=1}^{I}$, where the robustness is understood as the worst case approach, i.e. we will be looking for the following value:

$$
\operatorname{WCMAC}(\delta)=\min _{\underline{x}_{i} \in X} \max _{j=1, \ldots, J} \min _{x_{i j} \in \in\left[\underline{x}_{i}, \underline{x}_{i}+\delta\right]} \max _{i=1, \ldots I}\left|x_{i j}-x_{i}^{j}\right|
$$

where WCMAC $(\delta)$ stands for the worst case minimal adaptation cost and is based on the assumption that for each choice $\left\{\left[\underline{x}_{i i}, \underline{x}_{i}+\delta\right]\right\}_{i=1}^{I}\left(\delta_{i}\right.$ fixed) the worst case of the decision maker preference will occur, thus such $\mathbf{x}^{j_{0}}$ will be selected that $A C\left(\left\{\underline{x}_{i}\right\}_{i=1}^{I}, \mathbf{x}^{j_{0}}, \delta\right)$ will be maximal. E.g. in case of our example and the interval solution presented in Fig. 1 we assume that once we have selected this very solution and are prepared to implement any production/sales plan $\left(x_{1}, x_{2}\right)$ such that $x_{1} \in[2,3]$ and $x_{2} \in[1.5,2.5]$ (Fig. 1), it will turn out that the decision maker wants to implement the solution candidate $\mathbf{x}^{1}$ and we will have to incur the adaptation cost 1.5 (the worst case in Fig. 1). We are looking for such a solution $\left\{\left[\underline{x}_{i i}, \underline{x}_{i}+\delta\right]\right\}_{i=1}^{I}(\delta$ fixed) that the worst case adaptation cost will be as small as possible.

Finally, we propose to consider various values of $\delta$. It is clear than if $\delta$ is increased, the adaptation cost will not increase and it may decrease. Thus, a compromise has to found between the preparation cost (proportional to $\delta$, incurred before the decision maker preferences are known) and the adaptation cost (proportional, assuming the 
worst case situation, to $W C M A C(\delta)$ ), which may, but not has to be incurred in the future (it will have to be incurred if the decision maker whishes to have his preferred solution, but it is also possible that the decision maker will be more flexible, that he will accept something "closer" to our selected interval solution-than we will have to pay a bit less).

\section{Problem solution}

Problem (3), for a fixed value of $\delta$, can be formulated as a linear programming problem:

$$
\begin{aligned}
& \lambda \rightarrow \min \\
& \lambda \geq x_{i j}-x_{i}^{j}, \quad \lambda \geq-x_{i j}+x_{i}^{j}, \quad j=1, \ldots, J, \quad i=1, \ldots I \\
& \underline{x}_{i} \in X \\
& \underline{x}_{i}+\delta \in X \\
& \underline{x}_{i} \leq x_{i j} \leq \underline{x}_{i}+\delta
\end{aligned}
$$

This problem has $I \cdot J+I+1$ variables $\left(x_{i}^{j}\right.$ is not a variable, but a parameter with a given value, like $\delta$ ), thus the dimension of the problem depends strongly on the number of possible methods the decision maker might use to solve the multibjective problem, equal to I. The problem can be solved by means of the classical simplex algorithm.

Of course, we can also consider make $\delta$ change and minimize the sum of the cost of preparing the temporary solution $\left\{\left[\underline{x}_{i i}, \underline{x}_{i}+\delta\right]\right\}_{i=1}^{I}$ (proportional to $\delta$-thus we consider just the incremental cost of preparing an interval solution, we assume we are paying only for the width of the intervals corresponding to individual decision variables intervals, the cost of preparing a crisp solution will have to be incurred anyway and that is why it can be neglected) and the later, potential cost of adapting the solution $\left\{\left[\underline{x}_{i i}, \underline{x}_{i}+\delta\right]\right\}_{i=1}^{I}$ to the solution preferred by the decision maker. This will be a one objective problem with the objective function $W_{\delta} \delta+W_{\lambda} \lambda$, where values $W_{\delta}, W_{\lambda}$ would reflect, respectively, the unitary cost of preparing the solution of the "depth" $\delta$ (thus the solution $\left\{\left[\underline{x}_{i i}, \underline{x}_{i}+\delta\right]\right\}_{i=1}^{I}$ ) and the potential unitary cost of adapting this solution to the solution eventually preferred by the decision maker. The value $W_{\lambda}$ may be made a bit smaller than the actual unitary adaptation cost—as, first of all, this $\lambda$ corresponds to the worst case solution, and the reality may not be as bad as that, and secondly, even if the worst case occurs, the decision maker may, after maybe some negotiations, be happy with a solution closer to $\left\{\left[\underline{x}_{i i}, \underline{x}_{i}+\delta\right]\right\}_{i=1}^{I}$, somewhere between his preferred solution and the interval solution we have prepared, and then this cost would be smaller. Anyway, the choice of the values $W_{\delta}, W_{\lambda}$ depends on the decision maker.

\section{Example}

The example presented here is a continuation of the example from Sect. 2. We will solve the following problem for different values of $W_{\delta}, W_{\lambda}$, using STORM and its 
Table 1 Numerical results for the example

\begin{tabular}{lrllll}
\hline$W_{\delta}$ & $W_{\lambda}$ & $\underline{x}_{1}$ & $\underline{x}_{2}$ & $\delta$ & $\lambda$ \\
\hline 20 & 100 & 0,5 & 0,5 & 1,5 & 1 \\
100 & 20 & 2,25 & 2,25 & 0 & 1,75 \\
\hline
\end{tabular}

module "Linear programming", containing the simplex algorithm:

$$
\begin{aligned}
& W_{\delta} \delta+W_{\lambda} \lambda \rightarrow \min \\
& \lambda \geq x_{11}-x_{1}^{1}, \lambda \geq-x_{11}+x_{1}^{1} \\
& \lambda \geq x_{21}-x_{2}^{1}, \lambda \geq-x_{21}+x_{2}^{1} \\
& \lambda \geq x_{12}-x_{1}^{2}, \lambda \geq-x_{12}+x_{1}^{2} \\
& \lambda \geq x_{22}-x_{2}^{2}, \lambda \geq-x_{22}+x_{2}^{2} \\
& \underline{x}_{1} \leq x_{11} \leq \underline{x}_{1}+\delta \\
& \underline{x}_{2} \leq x_{21} \leq \underline{x}_{2}+\delta \\
& \underline{x}_{1} \leq x_{12} \leq \underline{x}_{1}+\delta \\
& \underline{x}_{2} \leq x_{22} \leq \underline{x}_{2}+\delta \\
& \underline{x}_{1} \leq 4 \\
& \underline{x}_{2} \leq 4 \\
& \underline{x}_{1}+\underline{x}_{2} \leq 6 \\
& \underline{x}_{1}+\delta \leq 4 \\
& \underline{x}_{2}+\delta \leq 4 \\
& \underline{x}_{1}+x_{2}+2 \delta \leq 6 \\
& \underline{x}_{1}, \underline{x}_{2} \geq 0
\end{aligned}
$$

The following results have been obtained for two selected couples $W_{\delta}, W_{\lambda}$.

In Table 1 we have the solution of problem (5) for selected values of $W_{\delta}, W_{\lambda}$. We can see that if the adaptation cots is comparatively higher $\left(W_{\delta}=20, W_{\lambda}=100\right)$, it is advantageous to prepare a proper interval solution, presented in Fig. 2, paying for it 30 monetary units, and being ready to pay additional 100 monetary units for the adaptation if the worst case occurs and the decision maker does not give up on his requirements. If we do not prepare such an interval solution, the total cost of implementing the final solution, equal to $20 \delta+100 \lambda$, risks to be higher than 130 - which is the minimal value of the objective function $20 \delta+100 \lambda$ in (5):

However, if the cost values are taken the other way round (the total cost in (5) is equal to $100 \delta+20 \lambda$ ), it is not profitable to prepare an interval solution, it is better to be ready just to implement a crisp solution $x_{1}=x_{2}=2,25$ and wait to see whether some adaptation will be necessary and to which extent. If the worst case really occurs, the 


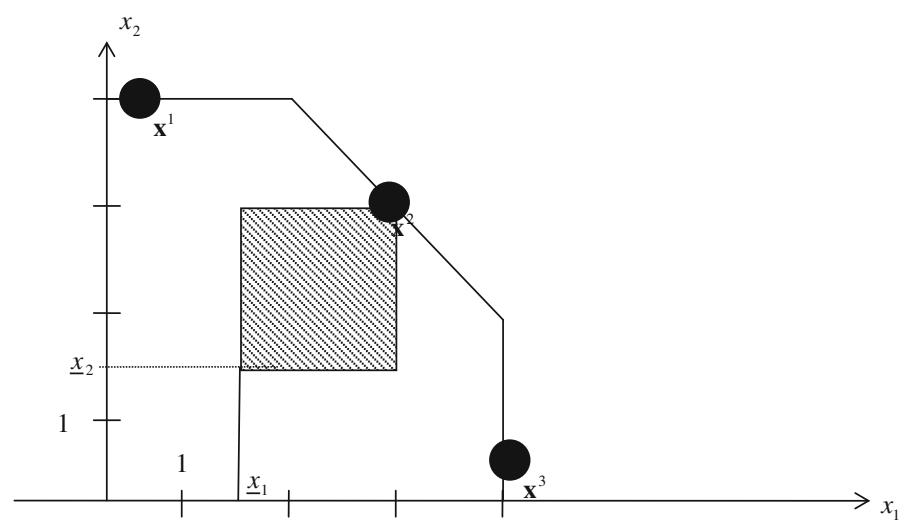

Fig. 2 The interval solution the company should be prepared to implement immediately in case we have $W_{\delta}=20, W_{\lambda}=100$

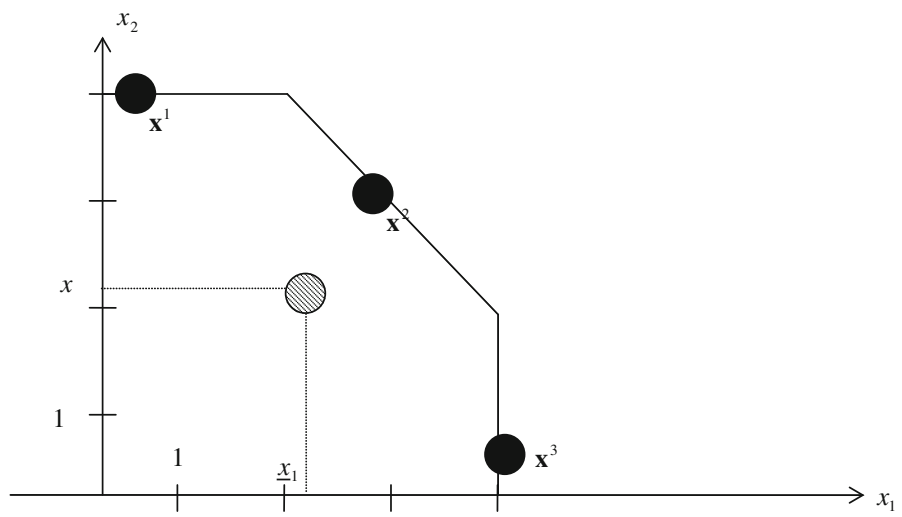

Fig. 3 The crisp solution the company should be prepared to implement immediately in case we have $W_{\delta}=100, W_{\lambda}=20$

adaptation would cost us 31 monetary units (and the preparation nothing-of course, the cost of preparing a crisp solution will actually be non-zero, but we have neglected it as something which will have to be incurred anyway and does not influence the decision) (Fig. 3 ).

\section{Conclusions}

We have proposed an approach to solve the multicriteria linear problem in which the eventual attitude of the decision maker towards the criteria is not fully known in the moment of decision making. We suggest to determine a kind of robust temporary solution, which will be a good starting point to implement the solution desired by the decision maker once his preferences become fully known. This solution will often be an interval one, because this will reduce the adaptation cost of the temporary solution 
to the final one. The problem can be solved by means of the simplex algorithm, thus does not involve any serious computational problems.

Open Access This article is distributed under the terms of the Creative Commons Attribution Noncommercial License which permits any noncommercial use, distribution, and reproduction in any medium, provided the original author(s) and source are credited.

\section{References}

Ben-Tal A, Nemirovsky A (1999) Robust solutions to uncertain programs. Oper Res Lett 25:1-13

El-Banna A-ZH, Zarea SA (2001) Stability of linear vector optimization problems corresponding to an efficient set. Math Comput Simul 57(6):335-345

Kouvelis P, Yu G (1997) Robust discrete optimization and its applications. Kluwer Academic Publishers, Boston

Kuchta D (2004) Robust goal programming. Control Cybern 33(3):501-510

Kuchta D (2010) A new concept of project plans robustness (in Polish). To be published in Modelowanie Preferencji i Ryzyko, Katowice Academy of Economics

Saad OM (1995) Stability on multiobjective linear programming problems with fuzzy parameters. Fuzzy Sets Syst 74(2):207-215

Steuer RE (1986) Multiple criteria optimization: theory, computation and application. Wiley, New York

Véronneau S, Cimon Y (2007) Maintaining robust decision capabilities: an integrative human-systems approach. Decision Support Syst 43(1):127-140

Vonder de SV, Demeulemeester EL, Herroelen WS, Leus R (2006) The trade-off between stability and makespan in resource-constrained project scheduling. Int J Prod Res 44(2):215-236 